\title{
Directed polymers in random media under confining force
}

\author{
Hyeong-Chai Jeong* \\ Department of Physics and Institute for Fundamental Physics, Sejong University, Seoul 143-747 \\ and Asia Pacific Center for Theoretical Physics, POSTECH, Pohang 790-784, Korea
}

(Received December 16, 2018)

\begin{abstract}
The scaling behavior of a directed polymer in a two-dimensional (2D) random potential under confining force is investigated. The energy of a polymer with configuration $\{y(x)\}$ is given by $H(\{y(x)\})=\sum_{x=1}^{N} \eta(x, y(x))+\epsilon W^{\alpha}$, where $\eta(x, y)$ is an uncorrelated random potential and $W$ is the width of the polymer. Using an energy argument, it is conjectured that the radius of gyration $R_{g}(N)$ and the energy fluctuation $\Delta E(N)$ of the polymer of length $N$ in the ground state increase as $R_{g}(N) \sim N^{\nu}$ and $\Delta E(N) \sim N^{\omega}$ respectively with $\nu=1 /(1+\alpha)$ and $\omega=(1+2 \alpha) /(4+4 \alpha)$ for $\alpha \geq 1 / 2$. A novel algorithm of finding the exact ground state, with the effective time complexity of $\mathcal{O}\left(N^{3}\right)$, is introduced and used to confirm the conjecture numerically.
\end{abstract}

\section{INTRODUCTION}

Scaling behaviors of a directed polymers in a random media (DPRM) have been studied extensively due to the mathematical interest of optimal path problems in random environment in addition to their application to physical systems such as stretched polymer in a gel or flux lines in a disordered superconductor [1]. A directed polymer is stretched in the longitudinal direction but can fluctuate in the transversal direction. We can map a configuration of a directed polymer in a $d$-dimensional space to a path of random walkers in the $d_{t}=d-1$ dimensional space (in the transversal direction) when the longitudinal direction is considered as the time. Therefore, in sufficiently low temperature, a DPRM corresponds to the minimum energy path of a random walker in a random potential $\eta(t, \vec{r})$. Its scaling properties are characterized by two exponents, transverse length scale exponent $\nu_{o}$ and energy fluctuation exponent $\omega_{o}$ [2]. For the $d_{t}=1$ dimensional walkers, the exact values of these exponents, $\nu_{o}=2 / 3$ and $\omega_{o}=1 / 3$ can be obtained analytically by the combinatorial method [3]. The exact exponent values can also be obtained by mapping the free energy of a random walker to Kardar-Parisi-Zhang equation (KPZ) via Cole-Hope transformation [1, 4, [5, 6, 7]. This mapping shows that the exponents for the DPRM, $\nu_{o}$ and $\omega_{o}$ should be equal to the inverse of the dynamic exponent, $1 / z_{K P Z}=2 / 3$ and the growth exponent, $\beta_{K P Z}=1 / 3$ of KPZ equation respectively at any finite temperature. In other words, any disorder drives a directed polymer in the $d=2$ dimensional media into a strong disorder, pinned phase at $T=0$.

In this paper, we study the scaling properties of a DPRM under confining force (DPRMCF). The Hamiltonian for a DPRMCF has two terms, the usual random potential term $E_{R M}$ of the DPRM and the confining energy term $E_{C}$ which prefers the straight polymer in global length scale. It may describe a DPRM confined by an in-

*Electronic address: hcj@sejong.ac.kr

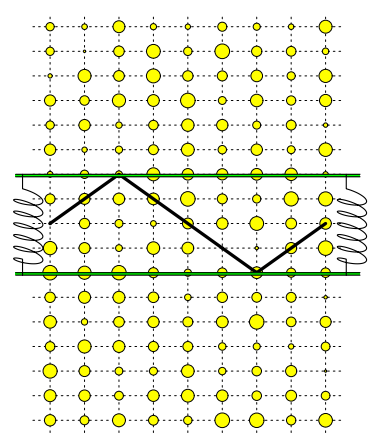

(a)

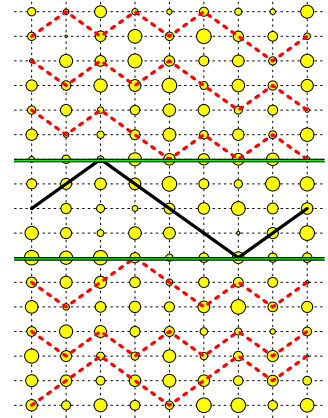

(b)
FIG. 1: (Color online) Directed polymers in 2D random media under confining force. The magnitude of the random potential at each lattice site is denoted by the area of the circles at the lattice site. A directed polymer is confined by the two rods. The confining force may come from the elasticity which are symbolized by the spring (a) or from the repulsion of the polymers (red dashed lines) at the outside of the rods (b).

flatable but non-flexible tube or by two rods with springs as illustrated in Fig. 11(a). We consider the confining energy which depends only on the "global width" $W$ of the polymer. Such confining energy term may mimic the elastic energy of the (inflatable) tube or the spring which prefers the smaller width. The confining force on the center polymer may arise from the repulsion from the other polymers (red dashed line) outside of the rods if we consider an array of polymers on a plate as illustrated in Fig. 1 (b). We may find other physical systems that our model might be applied, such as the motion of a single step on a terrace with quenched random impurities in a vicinal surface [8] but the principal motivation for the model is rather theoretical interest. The effects on the scaling properties of random walkers or directed polymers from the energy terms associated with the global configuration quantity, such as the global width $W$, are theoretically intriguing $[9,10,11,12]$. For example, the confining energy term $E_{c} \sim W$ makes the normal random walkers (without $E_{R M}$ ) visit the same $y$ value sites even 
times stochastically [9], and changes the "roughness" exponent to $1 / 3$ from the conventional universal value of $1 / 2$ 9, 12, 13]. The path of this stochastic even visiting random walker can be mapped a $1 \mathrm{D}$ interface profile which is called a "self-flattening" surface 10, 11]. The height-height correlation function of the self-flattening surface shows an anomalous scaling behavior in the sense that the local wandering exponent is different from the global roughness exponent 11]. This anomalous behavior indicates the existence of window length scale $l(N) \sim N^{\delta}$ with $0<\delta<1$ above which the global energy term becomes relevant. Recently, it has been conjectured that the window exponent $\delta$ is given by $\delta=\frac{D}{D+\nu_{o}}$ from an energy-entropy argument where $D$ is the dimension of the surface and $\nu_{o}$ is the roughness exponent without the selfflattening mechanism [14]. This argument seems to be valid for general self-flattening mechanism, which corresponds to non-zero finite temperature dynamics with the confining energy proportional to the width $W[10,11,12$.

Here, we study the scaling properties of the zero temperature, ground state paths for a DPRMCF in 2D with a confining energy in the form of $E_{c}=\epsilon W^{\alpha}$ 15]. If we assume the existence of window length $l(N) \sim N^{\delta}$, a power counting argument predict $\delta=3 /(2+2 \alpha)$ for $\alpha>1 / 2$ and the confining energy of any positive $\epsilon$ changes the roughness exponent $\nu$ to $1 /(1+\alpha)$ from $\nu_{o}=2 / 3$ and the energy fluctuation exponent $\omega$ to $(1+2 \alpha) /(4+4 \alpha)$ from $\omega_{o}=1 / 3$. This conjecture is numerically tested for a series of different $\alpha$ values.

This paper is organized as follows. In Sec. II, we define the DPRMCF model. An analytic prediction for the roughness exponent $\nu$ and the energy fluctuation exponent $\omega$ is presented in Sec. III. A novel algorithm for a numerical study of DPRMCF which finds the exact ground state in the effective time complexity of $\mathcal{O}\left(N^{3}\right)$ is introduced in Sec. IV. In Sec. V, we present the numerical results from a series of Monte Carlo Simulations for DPRMCF model and calculate the exponents $\nu$ and $\omega$. We conclude the paper with a summary and remarks in Sec. VI.

\section{MODEL}

We consider a discrete model for a directed polymer in a random media under confining force (DPRMCF) whose Hamiltonian has two terms, site random potential and confining energy. Before introducing the details of our DPRMCF model, let us first explain a discrete model for DPRM without the confining force. We consider polymers on a 2D lattice whose horizontal axis is $x$ and the vertical axis is $y$ (i.e., the $d_{t}=1$ dimensional transversal space is the $y$-axis). At each lattice site, random potential $\eta(x, y) \in[0,1]$ taken from a uniform distribution with

$$
\left\langle\eta(x, y) \eta\left(x^{\prime}, y^{\prime}\right)\right\rangle=\frac{1}{4}+\frac{1}{12} \delta_{x, x^{\prime}} \delta_{y, y^{\prime}}
$$

is assigned. Since overhangs are forbidden for a directed polymer, its configuration is specified by a single valued integer function $y(x) \in \mathbb{Z}$ with $|y(x+1)-y(x)|=1$. The energy of a DPRM is given by the sum of site potentials $\eta(x, y(x))$ on which the polymer lies. At zero temperature, a polymer on a given random potential locates at the path which minimizes the total site energies of the random media,

$$
E_{R M}(\{y(x)\})=\sum_{x=1}^{N} \eta(x, y(x)),
$$

where $N$ is the length of the polymer.

The configurational space of a DPRMCF is the same as that of a DPRM but the Hamiltonian $H$ of a DPRMCF has the confining energy term $E_{C}=\epsilon W^{\alpha}$ in addition to the $E_{R M}$,

$$
H(\{y(x)\})=\sum_{x=1}^{N} \eta(x, y(x))+\epsilon W^{\alpha} .
$$

Here, $W$ is the absolute "width" of the polymer defined by

$$
W=y_{\max }-y_{\min }+1,
$$

where $y_{\max }$ and $y_{\min }$ are the maximum and the minimum values of $y(x)$ respectively and $\epsilon$ and $\alpha$ are positive parameters so that the confining energy increases when the width grows.

\section{WINDOW HYPOTHESIS AND POWER COUNTING ARGUMENT FROM ENERGY FLUCTUATION}

We study the scaling properties of the zero temperature, ground state paths for the Hamiltonian of Eq. (3). The statistical behaviors of a polymer in random media are usually characterized by the radius of gyration $R_{g}$ and the energy fluctuation $\Delta E$ defined by

$$
\begin{aligned}
& R_{g}(N)=\sqrt{\left\langle\overline{(y-\bar{y})^{2}}\right\rangle} \\
& =\sqrt{\frac{1}{\Omega} \sum_{\{\eta\}}\left[\frac{1}{N} \sum_{x=1}^{N}\left(y_{\eta}(x)-\frac{1}{N} \sum_{x=1}^{N} y_{\eta}(x)\right)^{2}\right]}
\end{aligned}
$$

and

$$
\begin{aligned}
& \Delta E(N)=\sqrt{\left\langle(E-\langle E\rangle)^{2}\right\rangle} \\
& =\sqrt{\frac{1}{\Omega} \sum_{\{\eta\}}\left[H_{\eta}\left(\left\{y_{\eta}(x)\right\}\right)-\frac{1}{\Omega} \sum_{\{\eta\}} H_{\eta}\left(\left\{y_{\eta}(x)\right\}\right)\right]^{2}},
\end{aligned}
$$


where $\Omega$ is the number of different realizations of random potentials and $\left\{y_{\eta}(x)\right\}$ and $H_{\eta}\left(\left\{y_{\eta}(x)\right\}\right)$ are the ground state path and its energy for a given random potential $\eta$. These two quantities asymptotically increase as

$$
\begin{aligned}
R_{g}(N) & \sim N^{\nu} \\
\Delta E(N) & \sim N^{\omega}
\end{aligned}
$$

for the ground state paths for a variety of different distributions of the randomness [2, 16, 17].

In this section, we estimate $\nu$ and $\omega$ for the DPRMCF by comparing the $E_{R M}$ and $E_{C}$ in the ground state. For a directed polymer in a $2 \mathrm{D}$ random media, it has been well known that the energy of the minimum path of length $N$ can be asymptotically written as

$$
E_{D P R M}(N)=a N+b N^{\omega_{o}},
$$

with $N$ independent positive parameters, $a, b$ and $\omega_{o}$. As $N$ goes to infinity, the parameter $a$ becomes sample independent and is about 0.25 for a random potential $\eta(x, y) \in[0,1]$ taken from a uniform distribution. On the other hand, the second coefficient $b$ is a sample dependent parameter with a finite variation. The energy fluctuation of the DPRM with length $N$ is given by

$$
\begin{aligned}
\Delta E_{D P R M}(N) & =\sqrt{\left\langle\left(E_{D P R M}-\left\langle E_{D P R M}\right\rangle\right)^{2}\right\rangle} \\
& =\sqrt{(\Delta b)^{2}} N^{\omega_{o}},
\end{aligned}
$$

and $\omega_{o}$ is called the energy fluctuation exponent for the DPRM [5, 6].

We estimate $\nu$ and $\omega$ for our DPRMCF model using Eq. (9) with an assumption of the existence of "window" 9, 11]. The confining energy term is assumed to be relevant only beyond a certain length scale of $l \sim N^{\delta}$, so called "window" 9, 11] so that the polymer behaves like DPRM within the length scale of $l$. Then, the random potential energy $E_{R M}(l ; N)$ of DPRMCF with length $N$ can be written as

$$
\begin{aligned}
E_{R M}(l ; N) & =\left(a l+b l^{\omega_{o}}\right)(N / l) \\
& =a N+b N l^{\omega_{o}-1}
\end{aligned}
$$

and the total energy of DPRMCF is given by

$$
\begin{aligned}
H(l ; N) & =E_{R M}(N)+\epsilon W^{\alpha} \\
& =a N+b N l^{\omega_{o}-1}+c l^{\alpha \nu_{o}},
\end{aligned}
$$

where $a, b$ and $c$ are independent of $l$ and $N$ since the absolute width of the polymer $W$ is of order $l^{\nu_{o}}$. From the minimization of $H(l ; N)$ with respect to $l$ (i.e., from $\left.\frac{\partial H}{\partial l}=0\right)$, we have

$$
\alpha \nu_{o} \delta-\delta=1+\omega_{o} \delta-2 \delta
$$

and the window exponent $\delta$ and the roughness exponent $\nu$ are given by

$$
\begin{aligned}
\delta & =1 /\left(1+\alpha \nu_{o}-\omega_{o}\right) \\
& =3 /(2+2 \alpha), \\
\nu & =\delta \nu_{o} \\
& =\nu_{o} /\left(1+\alpha \nu_{o}-\omega_{o}\right) \\
& =1 /(1+\alpha),
\end{aligned}
$$

where we use $\nu_{o}=2 / 3$ and $\omega_{o}=1 / 3$ for 1D DPRM [2, 5, 6]. The above two equation for $\delta$ and $\nu$ are only valid for $\alpha \geq 1 / 2$ since $\delta$ cannot be larger than 1 . For $0 \leq \alpha<1 / 2$, we expect $\nu=\nu_{0}$ since the confining energy term is not relevant since the third term of Eq. (12) can be always neglected comparing to the second term as $N$ goes to infinity.

We can use a similar argument to estimate the energy fluctuation exponent. We first assume that the total energy fluctuation $\Delta H$ should show the same $N$ dependence as the random potential energy fluctuation $\Delta E_{R M}$ for $\alpha>1 / 2$ from the power counting argument. Then, for the estimation of $\Delta E_{R M}$, we use the same "window" argument used to extract the roughness exponent $\nu$. Since there are $N / l \sim N^{1-\delta}$ windows whose energies fluctuate with the "amplitude" $l^{\omega_{o}} \sim N^{\omega_{0} \delta}$, we have

$$
\Delta E_{R M}(N)=N^{\omega_{0} \delta} N^{\frac{1-\delta}{2}}
$$

and the energy fluctuation exponent $\omega$ is given by

$$
\begin{aligned}
\omega & =\omega_{o} \delta+\frac{1}{2}-\frac{1}{\delta} \\
& =\frac{1+2 \alpha}{4(1+\alpha)} .
\end{aligned}
$$

\section{EXACT ENUMERATION ALGORITHM}

In this section, we consider a numerical method to find the ground state of the DPRMCF. For a given random energy $\eta(x, y) \in[0,1]$ for the $2 \mathrm{D}$ lattice sites $(x, y) \in \mathbb{Z}^{2}$, we find the minimum energy path of Eq. (3) using an exact enumeration method under the constraint of $|y(x+1)-y(x)|=1$ with the anchored $(y(0)=0)$ boundary condition. Since the number of all possible paths increases as $2^{N}$ for a polymer of length $N$, it is (computationally) impossible for large $N$ (say, $N>50$ ) to find the ground state by comparing the energies of all paths. We present a novel, efficient algorithm of finding the exact ground energy path with the effective time complexity $\mathcal{O}\left(N^{3}\right)$, based on the transfer matrix algorithm (TMA) 2] for the DPRM problem. Figure 2(a) and (b) illustrate the TMA for the DPRM problem. We represent the random potential in (a) by writing its value at each lattice site in the circle at the site (For clarity, we show an integer-valued random potential $\eta(x, y) \in\{1,2, \ldots, 9\}$ instead of real numbers from $[0,1]$.) and show the minimum energy path in (b) by a solid line. The number in the circle at the site $(x, y)$ in (b) is the minimum potential energy $E_{m}^{0}(x, y)$ of a polymer from the origin to the 

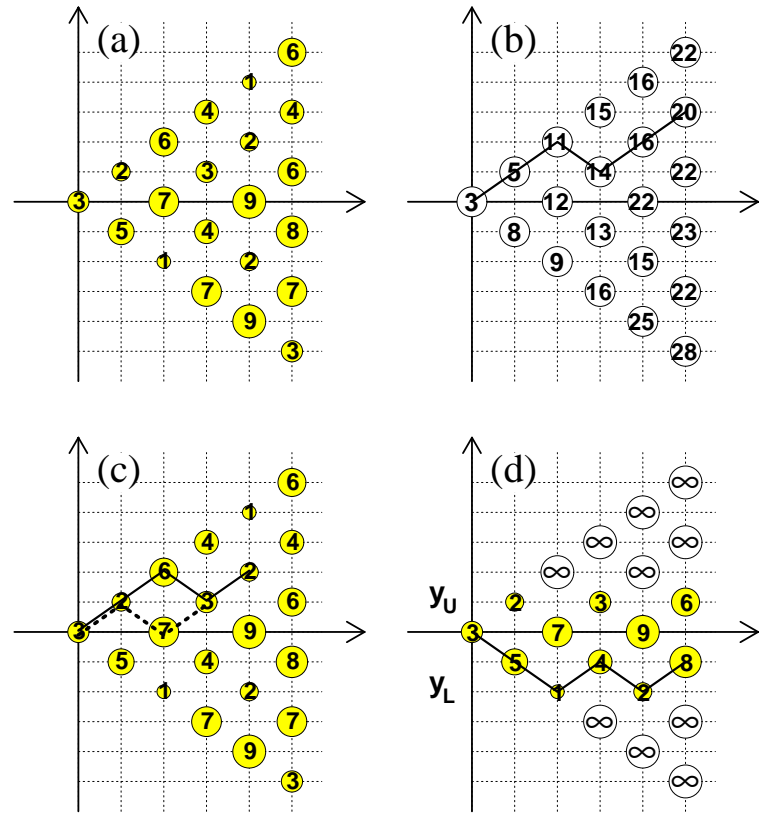

FIG. 2: Directed polymer in 2D random media. (a) A random potential is assigned at each lattice site. (b) Transfer matrix algorithm for a DPRM. The number in the circle at the site $(x, y)$ is the sum of site potentials of the minimum energy path from the origin to the site $(x, y)$. The solid line is the minimum energy path to $x=N$. (c) For the DPRMCF, the minimum energy path to the site $(x, y)$ is not a simple sum of the site $(x, y)$ and the minimum energy path to $(x-1, y-1)$ or $(x-1, y+1)$ (see text). (d) The minimum energy path for a modified potential $\tilde{\eta}_{y_{L}, y_{U}}(x, y)$ with $y_{L}=-2$ and $y_{U}=1$.

site $(x, y)$. The minimum energies $E_{m}^{0}(x, y)$ for all the $(x, y)$ with $|y|<x$ for $x \in\{1,2, \cdots, N\}$ can be obtained in $\mathcal{O}\left(N^{2}\right)$ time using the TMA,

$$
\begin{aligned}
E_{m}^{0}(x, y) & =\eta(x, y) \\
& +\min \left[E_{m}^{0}(x-1, y-1), E_{m}^{0}(x-1, y+1)\right]
\end{aligned}
$$

with $E_{m}^{0}(0,0)=\eta(0,0)$ and $E_{m}^{0}(x, y)=\infty$ for $|y|>x$, where $\min [A, B]$ is the minimum value of $A$ and $B$. The minimum energy path $P_{m}^{0}(x, y)$ from the origin to the site $(x, y)$ can be obtained similarly;

$$
P_{m}^{0}(x, y)=\left\{\begin{array}{l}
(x, y)+P_{m}^{0}(x-1, y-1) \\
\quad \text { if } E_{m}^{0}(x-1, y-1)<E_{m}^{0}(x-1, y+1) \\
(x, y)+P_{m}^{0}(x-1, y+1) \\
\quad \text { if } E_{m}^{0}(x-1, y-1)>E_{m}^{0}(x-1, y+1)
\end{array}\right.
$$

with $P_{m}^{0}(0,0)=(0,0)$. (If $E_{m}^{0}(x-1, y-1)=E_{m}^{0}(x-1, y+1)$, the minimum energy path is not unique and both paths become the minimum energy paths, but this is a very rare event for real values of $\eta$.) The ground state energy $E_{g}^{0}(N)$ is then given by the minimum of $E_{m}^{0}(N, y)$ over $y$,

$$
E_{g}^{0}(N)=\min _{y} E_{m}^{0}(N, y)
$$

for $|y| \leq N$ and the ground state path is given by

$$
P_{g}^{0}(N)=P_{m}^{0}\left(N, y_{m}^{0}(N)\right)
$$

where $y_{m}^{0}(N)$ is the site such that $E_{m}^{0}\left(N, y_{m}^{0}(N)\right)=$ $E_{g}(N)$. The ground state path for the random potential of Fig. 2(a) is obtained this way and shown in (b) as the solid line.

One might suppose that a similar transfer matrix algorithm, with the time complexity of $\mathcal{O}\left(N^{2}\right)$, might be possible for a DPRMCF. The first term of Eq. (18), $\eta(x, y)$ is the energy cost for a DPRM to proceed one step further from $x-1$ to $x$. A naive generalization of Eq. (18) is replacing $\eta(x, y)$ by $\eta(x, y)+\epsilon\left[(W+1)^{\alpha}-W^{\alpha}\right]$ when the one-step movement increases the polymer width from $W$ to $W+1$. However, such algorithm does not lead to the global ground state of Eq. (3) since the minimum energies $E_{m}(x-1, y-1)$ and $E_{m}(x-1, y+1)$ are not enough to determine $E_{m}(x, y)$ as illustrated in Fig. 2( $\mathrm{c}$ ) where $E_{m}(x, y)$ is the energy (including the confining term) of the ground state path to the point $(x, y)$ from the origin. As an example, consider the minimum energy path to the point $(4,2) P_{m}(4,2)$ for $\alpha=1$ and $\epsilon=2$ with $\eta(x, y)$ given in Fig. 2(a). By comparing energies of all paths, one can see that $P_{m}(4,2)$ is given by $P_{m}(4,2)=(0,0)-(1,1)-(2,2)-(3,1)-(4,2)$, denoted by the solid line in Fig. 2(c). This path is not a simple addition of the end point $(4,2)$ to $P_{m}(3,1)$ unlike the case of the DPRM. The minimum energy path to $(3,1)$ is given by $P_{m}(3,1)=(0,0)-(1,1)-(2,0)-(3,1)$, denoted by the dashed line. It has lower energy than $P_{e}(3,1)=(0,0)-(1,1)$ $(2,2)-(3,1)$ although $\eta(2,0)>\eta(2,2)$ since the global width of $P_{m}(3,1)$ is smaller than that of $P_{e}(3,1)$. However, $P_{m}(4,2)$ is given by the addition of the end point $(4,2)$ to $P_{e}(3,1)$ not to $P_{m}(3,1)$. This is because the global widths of both paths to $(4,2)$, the path through $P_{e}(3,1)$ and the path through $P_{m}(3,1)$, are the same as 3. Therefore, for a DPRMCF, we need information on the minimum height $y_{\min }$ and the maximum height $y_{\max }$ of the path as well as the potential energy of the path. In other words, the minimum energy $E_{m}(x, y)$ up to the site $(x, y)$ is not enough but we need to know the minimum energies $E_{m}\left(x, y, y_{\min }, y_{\max }\right)$ of the paths to $(x, y)$ for all different combinations of $y_{\min }$ and $y_{\max }$. The minimum energy $E_{m}\left(x, y, y_{\min }, y_{\max }\right)$ can be calculated from the minimum energies $E_{m}\left(x-1, y-1, y_{\min }^{\prime}, y_{\max }^{\prime}\right)$ and $E_{m}\left(x-1, y+1, y_{\min }^{\prime}, y_{\max }^{\prime}\right)$ for a proper combinations of $y_{\min }^{\prime}$ and $y_{\max }^{\prime}$ values and the ground state energy of Eq. (3), $E_{g}(N)$ is obtained as the minimum of $E_{m}\left(N, y, y_{\min }, y_{\max }\right)$, over $y, y_{\min }$, and $y_{\max }$,

$$
E_{g}(N)=\min _{y, y_{\min }, y_{\max }} E_{m}\left(N, y, y_{\min }, y_{\max }\right)
$$

In principle, one can construct an algorithm to find ground state energy and its path based on Eq. (22) but it generally requires $\mathcal{O}\left(N^{4}\right)$ memory and time. Although its "effective" time complexity can be reduced $\mathcal{O}\left(N^{3}\right)$ (see below), the $\mathcal{O}\left(N^{4}\right)$ memory requirement puts a strong 
upper bound on the sizes of the systems to be investigated.

In this paper, we use a novel algorithm which uses only $\mathcal{O}\left(N^{2}\right)$ memory but is more efficient than the algorithm using Eq. (22). As before, we imagine the set $Y_{y_{\min }, y_{\max }}$ of paths whose minimum and maximum heights are $y_{\text {min }}$ and $y_{\max }$ but we first consider the path with the minimum potential energy,

$$
E_{m}^{0}\left(N, y_{\min }, y_{\max }\right)=\min _{\{y(x)\} \in Y_{y_{\min }, y_{\max }}}\left[\sum_{x=1}^{N} \eta(x, y(x))\right]
$$

instead of the minimum total energy. Since the minimum total energy $E_{m}\left(N, y_{\min }, y_{\max }\right)$ for the given $y_{\min }$ and $y_{\max }$ values, is simply given by

$$
\begin{aligned}
E_{m}\left(N, y_{\min }, y_{\max }\right)= & E_{m}^{0}\left(N, y_{\min }, y_{\max }\right) \\
& +\epsilon\left(y_{\max }-y_{\min }+1\right)^{\alpha}
\end{aligned}
$$

the ground state energy $E_{g}(N)$ can be obtained by

$$
\begin{aligned}
E_{g}(N)= & \min _{y_{\min }, y_{\max }} E_{m}\left(N, y_{\min }, y_{\max }\right) \\
= & \min _{y_{\min }, y_{\max }}\left[E_{m}^{0}\left(N, y_{\min }, y_{\max }\right)\right. \\
& \left.\quad+\epsilon\left(y_{\max }-y_{\min }+1\right)^{\alpha}\right],
\end{aligned}
$$

from the minimum potential energies. Note that we always have $y_{\min } \leq 0$ and $y_{\max } \geq 0$ since we set $y(0)=0$ as the anchored boundary. Therefore, in general, we need to calculate $E_{m}^{0}\left(N, y_{\min }, y_{\max }\right)$ for $N^{2}$ different combination of $y_{\min } \in\{0,-1, \ldots,-N\}$ and $y_{\max } \in\{0,1, \ldots, N\}$ to get the ground state energy $E_{g}(N)$. However, for a confining energy with positive $\epsilon$ and $\alpha$, we do not have to look for the paths with $y_{\max } \geq W_{0}$ or $y_{\min } \leq-W_{0}$ where $W_{0}$ is the width of the minimum potential path $P_{g}^{0}$, which minimizes $E_{R M}$ of Eq. (2). Let $y_{\min }^{0}$ and $y_{\max }^{0}$ be the minimum and the maximum heights of the minimum potential path $P_{g}^{0}$. Then $E_{m}^{0}\left(N, y_{\min }^{0}, y_{\max }^{0}\right)$ is the minimum of the first term in the square bracket of Eq. (25) and $W_{0}=y_{\max }^{0}-y_{\min }^{0}+1$. The total energy of a path, whose $y_{\max } \geq W_{0}$ or $y_{\min } \leq-W_{0}$, cannot be smaller than that of $P_{g}^{0}$ since its confining energy is larger than $\epsilon W_{0}^{\alpha}$ in addition to the fact that its potential energy is lager than $E_{m}^{0}\left(N, y_{\min }^{0}, y_{\max }^{0}\right)$. Therefore, for a given $\eta(x, y)$, the ground state energy $E_{g}(N)$ of Eq. 25) can be obtained from the minimization of $E_{m}\left(N, y_{\min }, y_{\max }\right)$ over $y_{\min } \in\left\{0,-1, \ldots,-W_{0}+1\right\}$ and $y_{\max } \in\left\{0,1, \ldots, W_{0}-1\right\}$.

Now, let us introduce a simple way to calculate $E_{m}^{0}\left(N, y_{\min }, y_{\max }\right)$ for the $\left(y_{\min }, y_{\max }\right)$ pairs needed for the minimization of Eq. (25). We use the conventional TMA for the DPRM problem but with a series of modified site potentials,

$$
\tilde{\eta}_{y_{L}, y_{U}}(x, y)= \begin{cases}\eta(x, y) & \text { for } y_{L} \leq y \leq y_{U} \\ \infty & \text { otherwise }\end{cases}
$$

for $y_{L}>-W_{0}$ and $y_{U}<W_{0}$. Note that we cannot obtained the minimum energies $E_{m}^{0}\left(N, y_{\min }, y_{\max }\right)$ for all pairs of $\left(y_{\min }, y_{\max }\right)$ with $y_{\min }>-W_{0}$ and $y_{\max }<W_{0}$ by simply applying TMA to the potential $\tilde{\eta}_{y_{L}, y_{U}}$, since $\left(y_{\min }, y_{\max }\right)$ of the minimum potential path $P_{y_{L}, y_{U}}^{0}$ of $\tilde{\eta}_{y_{L}, y_{U}}$ is not necessary equal to $\left(y_{L}, y_{U}\right)$. As an example, Fig. 2(d) shows $P_{y_{L}, y_{U}}^{0}$ for $\left(y_{L}, y_{U}\right)=(-2,1)$. The minimum height of the $P_{y_{L}}^{0}, y_{U}$, $y_{\min }=-2$ is equal to $y_{L}$ but the maximum height of it, $y_{\max }=0$ is not equal to $y_{U}=1$. Therefore, the minimum path energies for the two modified random potentials $\tilde{\eta}_{-2,0}$ and $\tilde{\eta}_{-2,1}$ are the same as $E_{m}^{0}(N,-2,0)$ and we cannot obtain $E_{m}^{0}(N,-2,1)$ by the TMA with $\tilde{\eta}_{y_{L}, y_{U}}$. However, this means that $E_{m}^{0}(N,-2,1)$ is larger than $E_{m}^{0}(N,-2,0)$ and we can safely exclude $E_{m}(N,-2,1)$ from the candidates of the $E_{g}(N)$ since $E_{m}(N,-2,1)$ must be larger than $E_{m}(N,-2,0)$. In general, we cannot obtain $E_{m}\left(N, y_{L}, y_{U}\right)$ by the TMA with $\tilde{\eta}_{y_{L}, y_{U}}$ if $\left(y_{\min }, y_{\max }\right)$ of $P_{y_{L}}^{0}, y_{U}$ is not equal to $\left(y_{L}, y_{U}\right)$ but we can exclude $E_{m}\left(N, y_{L}, y_{U}\right)$ in the minimization of Eq. (25) since this means $E_{m}\left(N, y_{L}, y_{U}\right)$ is always larger than $E_{m}\left(N, y_{\min }, y_{\max }\right)$. If, the $\left(y_{\min }, y_{\max }\right)$ value is not equal to $\left(y_{L}, y_{U}\right), E_{m}^{0}\left(N, y_{L}, y_{U}\right)$ is larger than $E_{m}^{0}\left(N, y_{\min }, y_{\max }\right)$ in addition to $\left(y_{U}-y_{L}+1\right)^{\alpha}>$ $\left(y_{\max }-y_{\min }+1\right)^{\alpha}$. In other words, $E_{g}(N)$ can be obtained by finding the minimum of $E_{m}\left(N, y_{\min }, y_{\max }\right)$ over only the $\left(y_{\min }, y_{\max }\right)$ pairs which can be obtained by the TMA with $\tilde{\eta}_{y_{L}, y_{U}}$ for $y_{L}>-W_{0}$ and $y_{U}<W_{0}$.

Let us summary our algorithm to find the ground state for a DPRMCF. For a given $\eta(x, y)$, we first find the minimum potential path $P_{g}^{0}$ without the confining energy term by using the conventional TMA for a DPRM and calculate $W_{0}$. This can be done in $\mathcal{O}\left(N^{2}\right)$. Then we find $P_{y_{L}, y_{U}}^{0}$ by applying the TMA to a series of the modified potentials $\tilde{\eta}_{y_{L}, y_{U}}$ for $y_{L}>-W_{0}$ and $y_{U}<W_{0}$, and measure their potential energies $E_{m}^{0}\left(N, y_{\min }, y_{\max }\right)$ and widths $W=y_{\max }-y_{\min }+1$. For a given $y_{L}$ and $y_{U}$, $P_{y_{L}, y_{U}}^{0}$ can be obtained in time of order $N\left(y_{U}-y_{L}\right)$. Since $W_{0} \sim N^{2 / 3}$, all $P_{y_{L}}^{0}, y_{U}$ can be obtained in $\mathcal{O}\left(N^{3}\right)$ on average. The ground state energy $E_{g}(N)$ is then given by the minimization of $E_{m}\left(N, y_{\min }, y_{\max }\right)$ through Eq. (25) and the ground state path is given by the corresponding minimum energy path. Note that the ground state energies and their paths for all positive $\epsilon$ and $\alpha$ values can be obtained using Eq. 25) once we get $P_{y_{L}, y_{U}}^{0}$ for $y_{L}>-W_{0}$ and $y_{U}<W_{0}$.

\section{NUMERICAL RESULTS}

We first consider a harmonic confining energy of $\epsilon W^{2}$, that is, the $\alpha=2$ case with the random potentials $\eta(x, y) \in[0,1]$ taken from the uniform distribution. We simulate a random potentials $\eta$ using the computergenerated pseudo random numbers 18] and find the ground state path of Eq. (3) using the algorithm pre- 

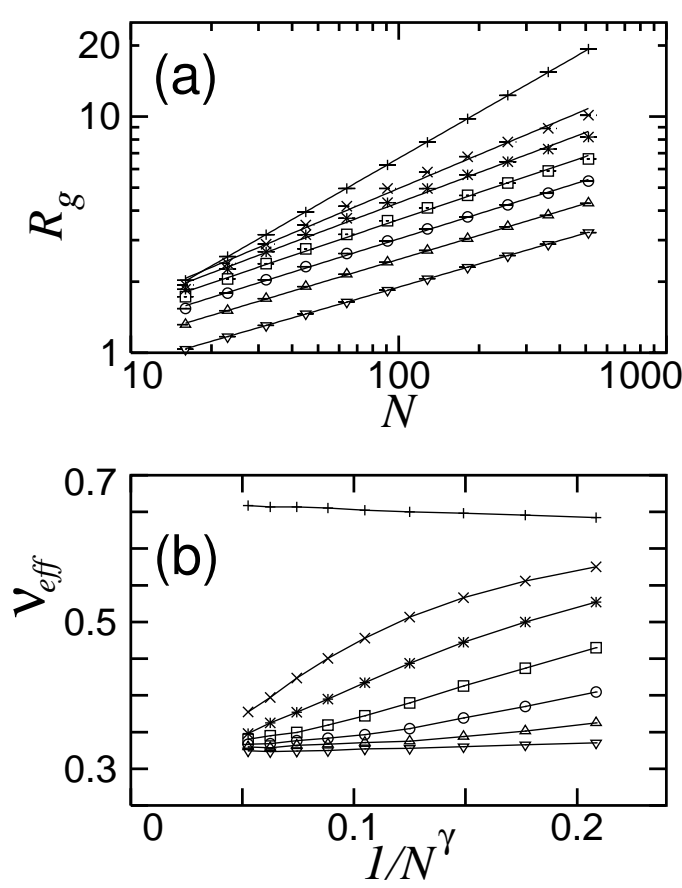

FIG. 3: (a) Radius of gyration $R_{g}$ for $N=16,23,32,45,64$, $92,128,256,362$, and 512 systems with a harmonic confining energy $E_{c}=\epsilon W^{2}(\alpha=2)$. From the top, $R_{g}$ for $\epsilon=0,0.001$, $0.002,0.004,0.008,0.016$ and 0.04 cases are shown. Fitting lines are in the form of $R_{g}=a N^{\nu}$ with $\nu=0.65,0.48,0.43$, $0.38,0.36,0.34$ and 0.33 respectively from the top. (b) Effective roughness exponents $\nu_{\text {eff }}$ for $\epsilon=0,0.001,0.002,0.004$, $0.008,0.016$ and 0.04 values are plotted against $1 / N^{\gamma}$ with $\gamma=0.5$ from the top. The uppermost curve of $\epsilon=0$ goes to the known value of $\nu_{o}=2 / 3$ but all the other curves go to much smaller value around $1 / 3$ as $N$ goes to infinity.

sented in the previous section. Then, we measure the square width $R_{\eta}^{2}$ (the square bracket of the Eq. (5) ) and the first $E_{\eta}$ and the second $E_{\eta}^{2}$ moments of the energy for the ground state path for the given $\eta$. The radius of gyration $R_{g}$ and $\Delta E$ are then obtained as $R_{g}=\sqrt{\left\langle R_{\eta}^{2}\right\rangle}$ and $\Delta E=\sqrt{\left\langle E^{2}\right\rangle-\langle E\rangle^{2}}$ where $\langle A\rangle$ mean the average over different realization of random potentials. We use eight million different realizations $\left(\Omega=8 \times 10^{6}\right)$ of random potentials for $N=16,23,32,45,64,92$ and 128 systems and four, two and one million different realizations for $N=256,362$ and 512 systems respectively to obtain the average values. The average over this large number of different random potentials makes the statistical error bars smaller than the sizes of the symbols most cases except the effective energy fluctuation exponents œ shown in Fig. 4(b) and Fig. 7 (d) later.

Figure 3 (a) shows the radius of gyration $R_{g}$ for the harmonic confining energy $E_{c}=\epsilon W^{2}$ as a function of polymer length $N$ for $\epsilon=0,0.001,0.002,0.004,0.008,0.016$, and 0.04 cases. For each $\epsilon$ value, $R_{g}$ lies on a straight line in a $\log -\log$ scale plot indicating $R_{g}(N) \sim N^{\nu}$. The least $\chi^{2}$ fits of $R_{g}(N) \sim N^{\nu}$ give $\nu=0.66,0.48,0.43,0.38,0.36$, 0.34 and 0.33 for $\epsilon=0,0.001,0.002,0.004,0.008,0.016$, and 0.04 cases respectively. The "roughness" exponents for $\epsilon>0.04$ are almost identical to those of the $\epsilon=0.04$ case unless $\epsilon$ is very large where the finite size effect is strong.

To estimate $\nu$ values for $N \rightarrow \infty$, we calculate $N$ dependent effective roughness exponent $\nu_{\text {eff }}$ defined by the successive slopes in the $\log -\log$ plot. We use neighboring three points to get the local slope, i.e., $\nu_{e f f}\left(N_{k}\right)$ is obtained as the slope of the least $\chi^{2}$ fit using the three data $R_{g}\left(N_{k-1}\right), R_{g}\left(N_{k}\right)$, and $R_{g}\left(N_{k+1}\right)$, where $N_{k}$ are the system sizes in an ascending order, $16=N_{1}<N_{2}<$ $\cdots<N_{10}=512$. The roughness exponent $\nu$ is estimated by extrapolating $\nu_{\text {eff }}$ in the infinite size limit with a plot $\nu_{\text {eff }}$ against $1 / N^{\gamma}$. In Fig. [3(b), we choose $\gamma=0.5$ which characterizes the trend of $\nu_{\text {eff }}$ for large $N$ well. From the figure, we see that our numerical measurement of $\nu$ for $\epsilon=0$ is consistent with the known value $\nu_{o}=2 / 3$ of the DPRM. However, it is clear that the roughness exponents of the DPRMCF $(\epsilon>0)$ is not equal to $\nu_{o}$. It is difficult to extract the definite $\nu$ values from our numerical data but we speculate that $\nu_{\text {eff }}$ goes to the conjectured value of $1 / 3$ for all $\epsilon>0$ as the system size $N$ goes to infinity.

Figure 4 (a) shows the energy fluctuation $\Delta E$ as a function of polymer length $N$ for the above seven $\epsilon$ values. As in the case of $R_{g}(N)$, for each $\epsilon$ value, $\Delta E$ lies on a straight line in a $\log -\log$ scale plot indicating a power law increase. The least $\chi^{2}$ fits of $\Delta E(N) \sim N^{\omega}$ give $\omega=0.32,0.36,0.37,0.39,0.40,0.41$ and 0.41 for $\epsilon=0$, $0.001,0.002,0.004,0.008,0.016$, and 0.04 cases respectively. We estimate the energy fluctuation exponent $\omega$ as the $N \rightarrow \infty$ limit of the effective exponents $\propto$ as for the roughness exponent. The effective exponent $œ$ is defined

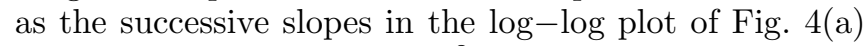
and obtained by the least $\chi^{2}$ fit with the three neighboring points. In Fig. 廿(b), the effective exponents are plotted against $1 / N^{\gamma}$ with $\gamma=0.5$ as before. From the figure, we see that our numerical measurement of $\omega$ for $\epsilon=0$ is consistent with the known value $\omega_{o}=1 / 3$ of the DPRM. For $\epsilon \neq 0$, all curves seem to go the conjectured value of $\omega=\frac{5}{12} \approx 0.417$.

In addition to the radius of gyration, we also measure the height (transverse position) correlation function $G(x ; N)$ to check the "window" argument. The correlation function $G(x ; N)$ is defined by the mean square height of the site $x$ of a polymer of length $N$,

$$
\begin{aligned}
G(x ; N) & =\left\langle y(x)^{2}\right\rangle \\
& =\frac{1}{\Omega} \sum_{\{\eta(x, y)\}} y_{\eta}^{2}(x)
\end{aligned}
$$

where $\Omega$ and $y_{\eta}(x)$ are the same as in the Eq. (5).

Figure [5(a) shows the $G(x ; N)$ for the DPRM $(\epsilon=0)$ for $N=32,64,128,256$ and 512 systems. All the data collapse to a single curve with $G(x) \sim x^{2 / 3}$ except the 

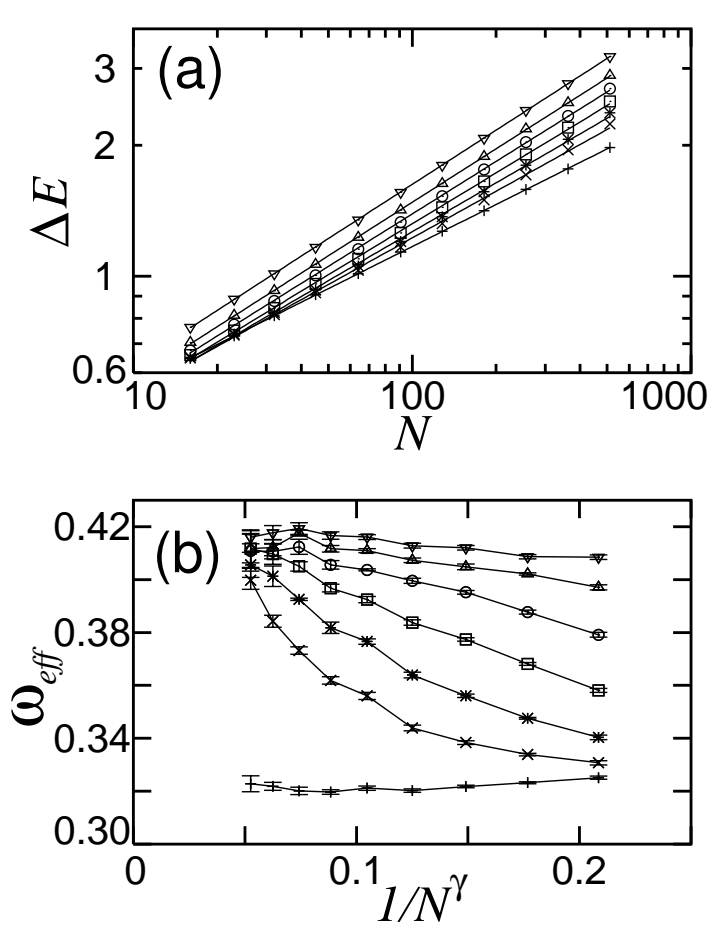

FIG. 4: (a) Measured energy fluctuation $\Delta E$ for the systems of sizes $N=16,23,32,45,64,92,128,256,362$, and 512 for $\alpha=2$. From the bottom, $\Delta E$ for $\epsilon=0,0.001,0.002,0.004$, $0.008,0.016$ and 0.04 cases are shown. Fitting lines are in the form of $\Delta E=a N^{\omega}$ with $\omega=0.32,0.36,0.37,0.39,0.40$, 0.41 and 0.41 respectively from the bottom. (b) Effective exponents œ for the above 7 different values of $\epsilon$ are plotted against $1 / N^{\gamma}$ with $\gamma=0.5$. As $N$ goes to infinity, the lowest curve of $\epsilon=0$ goes to the known value of $\omega_{o}=1 / 3$ but all the other curves go to much larger value around the predicted $\omega$ of $5 / 12$.

points near or at the boundaries at $x=N$. Note that the ground state path $y_{\eta}(x)$ for a given random potential $\eta$ cannot be determined locally even in the case of $\epsilon=$ 0 . In other words, the minimum energy path up to $x$ is different from the sub-path up to $x$ of the minimum energy path of length $N>x$. Due to the global nature of the ground state path determination, $G(x ; N)$ deviate form the infinite size behavior for a finite portion of $N$ even for the free boundary condition [19]. Yet, the height correlation function follows the scaling relation,

$$
G(x ; N)=N^{2 \nu_{o}} g_{o}(x / N)
$$

where $g_{o}(u)$ increases as $g_{o}(u) \sim u^{2 \nu_{o}}$ unless $u$ is very close to 1 where the boundary effect exists. When we rescale the correlation function $G(x ; N)$ by $N^{2 \nu_{o}}$ with $\nu_{o}=2 / 3$ and $x$ by $N$, all the data collapse to a single

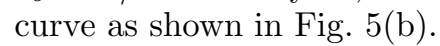

However, the correlation function $G(x ; N)$ for $\epsilon>0$ shows qualitatively different behaviors from the DPRM
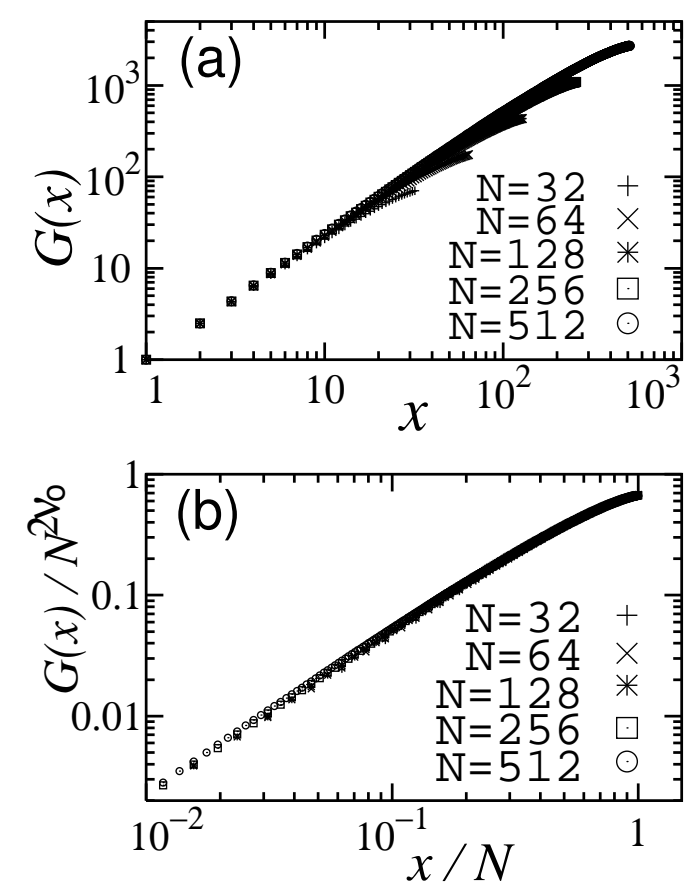

FIG. 5: (a) $G(x)$ for the systems of sizes $N=32,64,128$, 256 , and 512 with no confining force $\epsilon=0$. (b) A rescaled height correlation $G(x) / N^{2 \nu_{0}}$ is plotted against rescaled distance $x / N$ with $\nu_{0}=2 / 3$ in log scale.

case. When there is an energy term associated with the global width, there seems to be another length scale over which the correlation function saturated. Figure [6(a) shows the $G(x ; N)$ for $\epsilon=0.04$ for $N=32,64,128,256$ and 512 systems. As $x$ increases, the correlation functions increase algebraically only for $x \leq l(N)$ and then remains as constant values. We rescale the correlation functions $G(x ; N)$ by $N^{2 \nu}$ and $x$ by $N^{\delta}$ with the conjectured values of Eq. (15), $\nu=1 / 3$ and $\delta=1 / 2$ and plot $G(x ; N) / N^{2 \nu}$ against $x / N^{\delta}$ in Fig. 6(b). All data collapse to a single curve, implying a new scaling law

$$
G(x ; N)=N^{2 \nu} g\left(x / N^{\delta}\right) .
$$

The scaling function $g(u)$ increases as $g(u) \sim u^{2 \nu_{o}}$ for $u<1$ and then becomes a constant for $u>1$. In other words, $G(x ; N)$ increase as $x^{2 \nu_{0}}$ for $x<N^{\delta}$ and reaches a constant value for $x>N^{\delta}$. Note that the scaling function grows algebraically with exponent $2 \nu_{0}$ not with $2 \nu$ supporting the assumption that our polymer behaviors like a DPRM up to the window size $N^{\delta}$ and then feels the global constraints of the confining force over the window size.

We perform a series of simulations for other values of $\alpha$ and obtain the numerically results consistent with the conjecture of Eq. (15). Figure 7 shows the radius of gyration $R_{g}$ and the energy fluctuation $\Delta E$ and their effective exponents $\nu_{\text {eff }}$ and $\propto$ for the confining energy of 

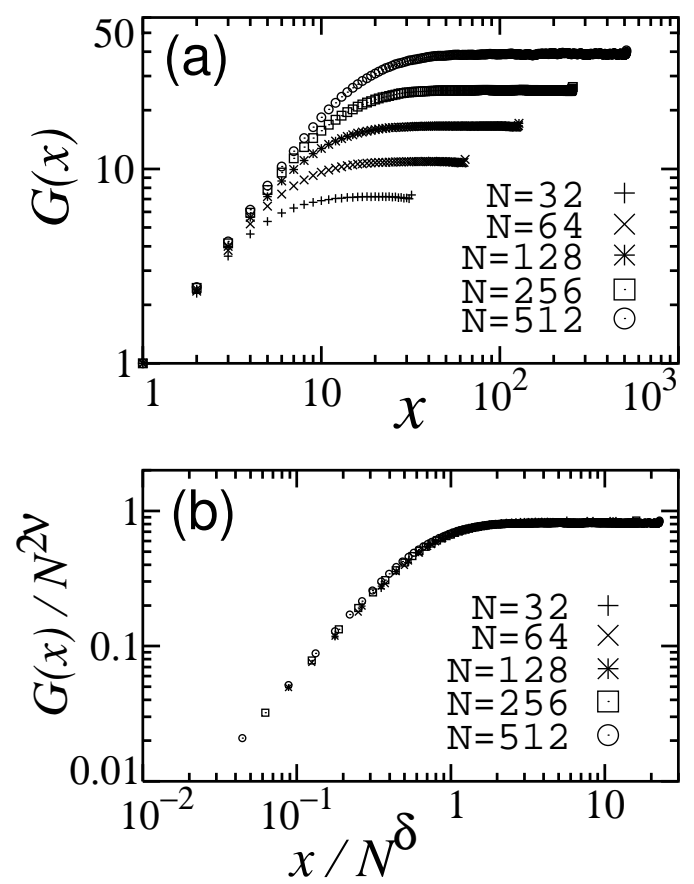

FIG. 6: (a) The correlation function $G(x)$ for the systems of sizes $N=32,64,128,256$, and 512 with a confining energy $E_{c}=0.04 W^{2}$. (b) The rescaled height correlation $G(x) / N^{2 \nu}$ is plotted against rescaled distance $x / N^{\delta}$ in a log scale with $\nu=1 / 3$ and $\delta=1 / 2$.

$W_{c}=\epsilon W(\alpha=1)$. The radius of gyration $R_{g}$ lies on a straight line in a $\log -\log$ scale plot as before and the least $\chi^{2}$ fits of $R_{g}(N) \sim N^{\nu}$ give $\nu=0.65,0.57,0.53,0.49,0.46$ and 0.45 for $\epsilon=0,0.05,0.1,0.2,0.5$, and 1.0 respectively. Note that the $\nu$ values from the least $\chi^{2}$ fits (using $R_{g}$ for $N \leq 512$ ) are smaller than the conjectured value of $1 / 2$ for some large epsilon values while those for small epsilon values are larger than the conjectured values unlike the case of $\alpha=2$. Yet, as the system size goes to infinity, all the effective roughness exponents $\nu_{\text {eff }}$ seem to go to the conjectured value of $1 / 2$ for $\epsilon>0$ while $\nu_{\text {eff }}$ for $\epsilon=0$ goes to the known value of $2 / 3$ (see Fig. Z (b)). The energy fluctuation $\Delta E$ is also measured and the effective energy fluctuation exponents $œ$ are calculated from them. As shown in Fig. Z( (c), the energy fluctuation $\Delta E$ increases as $\Delta E \sim N^{\omega}$ with $\omega=0.32,0.33,0.34,0.35,0.35$ and 0.35 for $\epsilon=0,0.05,0.1,0.2,0.5$, and 1.0 respectively. From these data, it seems to be difficult to distinguish the energy fluctuation exponent of DPRMCF from that of DPRM. The analysis of the effective exponents $\nu_{e f f}(N)$ provides somewhat better diagnosis. In Fig. Z7(d) we plot $\nu_{\text {eff }}$ against $1 / N^{0.5}$ as before. The error bars of œ for $\alpha=1$ are relatively large as shown in the figure although we obtain the effective exponent with more than million different random potentials. The statistical error of one millionth in measuring the second moment average $\left\langle E^{2}\right\rangle$
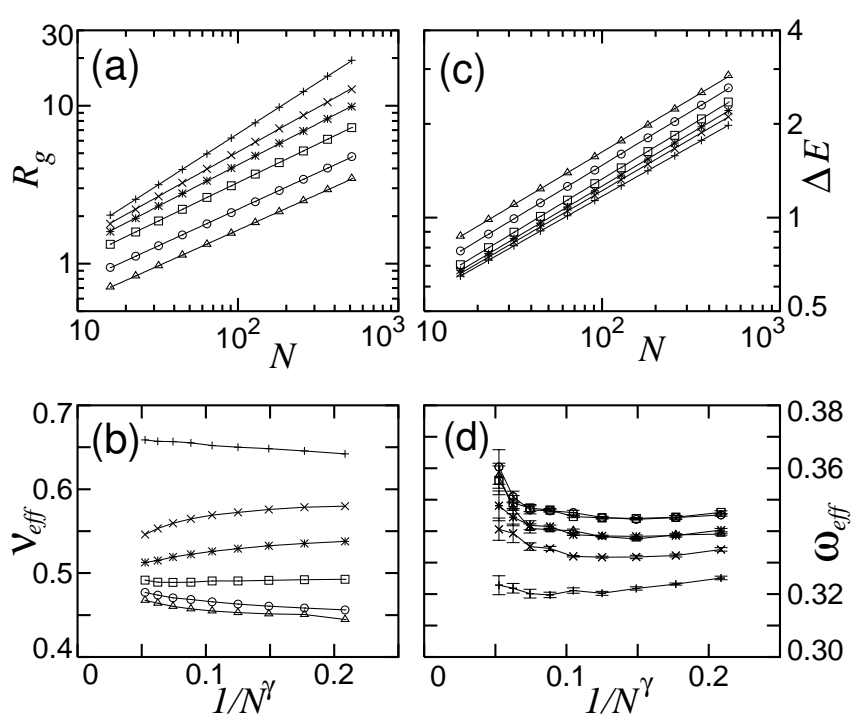

FIG. 7: (a) Measured $R_{g}$ for the systems of sizes $N=16,23$, $32,45,64,92,128,256,362$, and 512 for $\alpha=1$. From the top, $R_{g}$ for $\epsilon=0,0.05,0.1,0.2,0.5$, and 1.0 cases are shown. Fitting lines are in the form of $R_{g}=a N^{\nu}$ with $\nu=0.65,0.57$, $0.53,0.49,0.46$ and 0.45 from the top. (b) Effective roughness exponents $\nu_{\text {eff }}$ for the above 7 different values of $\epsilon$ are plotted against $1 / N^{\gamma}$ with $\gamma=0.5$. As the system size goes to infinity, the effective exponents seems to go to the conjectured value 0.5 for all $\epsilon \neq 0$. (c) Energy fluctuations $\Delta E$ are plotted against $N$. Fitting lines are in the form of $\Delta E=a N^{\omega}$ with $\omega=0.32,0.33,0.34,0.35,0.35$ and 0.35 in an ascending order or epsilon values from the bottom. (d) Effective energy fluctuation exponents $\propto$ against $1 / N^{\gamma}$ with $\gamma=0.5$. The lowest curve is for the $\epsilon=0$ case.

gives rise to the error bars of the symbol size in Fig. 17(d). Due to these limitations, we cannot extract the definite value of $\omega$ for $\epsilon>0$ from our simulations but the numerical results does not seem to exclude the conjectured value of $\omega=3 / 8=0.375$. Our simulations on other $\alpha$ values such as $\alpha=1.5$ and $\alpha=3$ also give the consistent results with the conjecture of Eqs. (15) and (17).

\section{CONCLUDING REMARKS}

We consider the scaling behavior of a directed polymer in a $2 \mathrm{D}$ random media with confining energy $E_{c}=\epsilon W^{\alpha}$ and find that the roughness exponent $\nu$ and the energy fluctuation exponent $\omega$ are given by $\nu=1 /(\alpha+1)$ and $\omega=(2 \alpha+1) / 4(\alpha+1)$ respectively. These results can be understood by assuming that a polymer of length $N$ behaves like a DPRM up to the window size $l(N) \sim N^{\delta}$ and then feels the confining energy over the window size.

We have only considered the scaling behavior of the zero temperature ground state polymers. We know the finite temperature behaviors of the polymers only for some 
limiting cases. For DPRM where confining energy term is absent, zero temperature pinned phase is the fixed point so that polymers at any finite temperature shows the same exponents with those at the zero temperature. On the other hands, if there is only confining energy (without random potential) there are three phases as temperature changes. At zero temperature, the polymer becomes a straight line with width 1 and therefore $\nu=0$ while it becomes a random walk with $\nu=1 / 2$ at infinite temperature. At nonzero finite temperature, especially for $\alpha=1$ where the confining energy is given by $E_{c}=\epsilon W$, the polymer configurations are identical to the self flattening surface [9] whose roughness exponent $\nu$ is $1 / 3$. When there are both random potential and confining energy terms, we do not know the scaling behaviors of the polymers at finite temperature where our algorithm cannot be applied. Further investigations are needed to explore full phase diagram of DPRMCF over general temperature and dimensions.

\section{Acknowledgments}

We thank J. M. Kim, J. D. Noh and H. Choi for useful comments and KIAS for the hospitality during the visit. This work was supported by the Korea Research Foundation Grant (KRF-2004-015-C00188) and the numerical calculations in this work were performed by using the supercomputing resources of Korea Institute of Science and Technology Information.
[1] A. L. Barabási and H. Stanley, Fractal Concepts in Surface Growth (Cambridge, New York, 1995).

[2] D. A. Huse and C. L. Henley, Phys. Rev. Lett. 54, 2708 (1985).

[3] K. Johansson, Commun. Math. Phys. 209, 437 (2000).

[4] M. Kardar and Y. C. Zhang, Phys. Rev. Lett. 58, 2087 (1987).

[5] J. M. Kim, M. A. Moore, and A. J. Bray, Phys. Rev. A 44, 2345 (1991).

[6] J. Krug, P. Meakin, and T. Halpin-Healy, Phys. Rev. A 45, 638 (1992).

[7] T. Halpin-Healy and Y. C. Zhang, Phys. Rep. 254, 217 (1995).

[8] H.-C. Jeong and E. D. Williams, Surface Science Reports 34, 171 (1999).

[9] J. D. Noh, H. Park, D. Kim, and M. den Nijs, Phys. Rev. E 64, 046131 (2001).

[10] Y. Kim, S. Y. Yoon, and H. Park, Phys. Rev. E 66, 040602(R) (2002).

[11] H.-C. Jeong, J. M. Kim, H. Choi, and Y. Kim, Phys. Rev. E 67, 046117 (2003).

[12] H.-C. Jeong and J. M. Kim, Phys. Rev. E 66, 051605 (2002).

[13] C.-H. Kim, H.-C. Jeong, and J. M. Kim, J. Korean Phys. Soc. 44, 547 (2004).

[14] H. Park, Phys. Rev. E 68, 053601 (2003).

[15] Note that our confining energy $E_{c}=\epsilon W^{\alpha}$ depends only on the global width of the interface and is different from the usual power-law confining potentials considered in "complete wetting" where the potentials are given as functions of local heights.

[16] A. Hartmann and H. Rieger, Optimization Algorithms in Physics (Wiley-VCH, Berlin, 2001).

[17] A. Hansen and J. Kertesz, Phys. Rev. Lett. 93, 040601 (2004).

[18] To check the possibility of the systematic errors from the computer generated pseudo random numbers, we use four different random number generators, drand 48 in the standard library and ran1, ran2 and ran3 in the numerical recipes for the simulations for the systems with $N \leq 128$. With each random number generator, we perform two million different simulations and confirm that all the measured quantities including $R_{g}, \nu_{e f f}, \Delta E$, and $\omega_{\text {eff }}$ coincide within the statistical errors. For $N>128$, we use drand 48 .

[19] When the path configurations are locally determined as in a restricted solid on solid model (RSOS), the boundary effects on the height correlation function $G(x ; N)$ exist only for the sites in a finite range from the boundary $(N-x<C)$ unless the boundary condition puts a global constraint. For example, $G(x ; N)$ of a RSOS model with the free boundary condition has no finite size effect [1] unlike that with the periodic boundary condition where the global sum of the local slops must be zero. 